\title{
Evaluation of new reimbursement fee for care of pharmacists in Japan
}

\begin{abstract}
There were so many criticisms of the pharmacy services that were reimbursed from the National Health Services in Japan. In order to overcome to this criticism and endorse the pharmacies and pharmacists to play pivotal functions in Japanese aging society, the Ministry of Health, Labor and Welfare (MHLW) published "Pharmacy Vision for Patients" and introduced the Your Pharmacist Reimbursed Program. On October of 2015, the MHLW published "Pharmacy Vision for Patients". The vision endorsed that pharmacist dispenses the patient medications unitarily according to all of the patient prescriptions from every hospitals and clinics. On the revision of reimbursement program in 2016, which included establish the new reimbursement fee "Your Pharmacist Reimbursed Program" was conducted in order to make the vision would be put into practice. These actions have given tremendous impact for Japanese pharmacists and pharmacies. In this manuscript, we introduced the YPRP and evaluate the impact of YPRP that affect the care of pharmacists and behavior of patients using the public data from Central Social Insurance Medical Council which is the government council body organizing the Japanese medical insurance program.
\end{abstract}

Introducing the YPRP has seen that relationship between pharmacists and patients have been closer and improved, and also pharmacists could provide medication therapy management to the patients continuously and exclusively. While for patients, change in consciousness has been founded that expectation for family pharmacists is growing that family pharmacists could observe all taken medications for each patient. It seems that the YPRP gave good effect and impact to the medication therapy in Japan. The MHLW has announced the new policies to enhanced pharmacists, pharmacies to play the pivotal role for medication therapy management of patients in Japanese aging society. Japanese pharmacies/pharmacists have to change the function and role of pharmacy to meet patient needs and demands from public society with using the new policies which is introduced by the MHLW. Furthermore, pharmacies/pharmacists have to use the newly established YPRP in careful manner and improve the satisfaction of patients, and then, pharmacies/ pharmacists have to step up to the next stage to adapt to the progressive aging society by expanding and developing the YPRP.

Keywords: reimbursement for pharmacy/pharmacists, vision for pharmacy, impact of the your pharmacist reimbursed program, Japan
Volume 6 Issue 4 - 2018

\author{
Kiyohito Nakai,' Atsushi Kawahara, ${ }^{2}$ Miwako \\ Kamei, ${ }^{2}$ Naomi Kurata' \\ 'School of Pharmacy, Showa University, Japan \\ ${ }^{2}$ School of Pharmacy, Nihon University, Japan
}

Correspondence: Kiyohito Nakai, Director of Medical Device Evaluation Division, Ministry of Health, Labor and Welfare, I-22, Kasumigaseki, Chiyodaku, Tokyo I0089I6 Japan,

Email nakaikiyohito@hotmail.com, nakai-kiyohito@mhlw.go.jp

Received: July 25, 2018 | Published: August 09, 2018

\section{Introduction}

We sometimes faced criticisms of the pharmacy services that were reimbursed from the National Health Services in Japan. One of the criticisms were that some lobbyist argued the pharmacy services were inadequate for the fee of pharmacy and also that pharmacy chain owner got the huge salary such as more than million dollars. In Japan, there are pharmacies located in front of the hospital that called as "Monzen Pharmacy". The word of "Monzen" means originally being located in front of a popular temple or shrine in order to give services such as restaurant/souvenir shops specialized for the visitors. In Japan, many patients who visit the hospital will go to each Monzen pharmacy to get dispensed medicines. In the Monzen pharmacy, their dispensing operation is generally very quick and not for patients, but is efficient as the pharmacy business. As the result, the Monzen pharmacy is subjected to big criticisms that they just dispense the medicine mechanically according to the prescription and hard criticisms that the Monzen pharmacies work not for the patients but for the specific hospital.

Aging society has been progressed in Japan, so that it is projected that one in four is an elderly person and that demands of medical and nursing care show a marked increase in the near future. It is said that we have to established the new community system to realize every person can live a life with dignity even in the progressive aging society by 2025 when the demands of medical and nursing care will be jumped up caused by the baby boom generation will reach their age of 75 . In this aging society, the function and role of pharmacies/pharmacists to be different from the current ones are expected. Therefore, pharmacies/ pharmacists have to find their appropriate function and role, and also have to adapt themselves smoothly to the progressive aging society even though no one in the world has an experience. The Ministry of Health, Labor and Welfare (MHLW) published "Pharmacy Vision for Patients" that intended to remind all of the pharmacy original aim and function for patients, and drive them to convert their current model to the family pharmacy model on October of $2015 .^{1}$

The "Pharmacy Vision for Patients" endorsed that pharmacist dispenses the patient medications unitarily according to all of the patient prescriptions from every hospitals and clinics where patients visit in order that the pharmacists may hold the patients' drug history and its related health history, and then the pharmacists could provide the most appropriate medication therapy management to the patient. The MHLW has also introduced the Your Pharmacist Reimbursed Program (YPRP) in the National Health Services to give incentives for the pharmacies to implement the functions described in the 
Pharmacy Vision for Patients. These actions have given tremendous impact for Japanese pharmacists and pharmacies. In this manuscript, we introduced the YPRP and evaluate the impact of YPRP that affect the care of pharmacists and behavior of patients using the public data from Central Social Insurance Medical Council which is the government council body organizing the Japanese medical insurance program.

\section{Revision of reimbursement for pharmacy}

The MHLW announced that the MHLW will endorse the pharmacies to implement function and services described in the "Pharmacy Vision for Patients" by utilizing opportunity of the revision of reimbursement program for pharmacy. On the revision of reimbursement program in 2016, the first step of fundamental and structural reimbursement reform for pharmacy which included establish the new reimbursement fee and weighting the fee for counseling the patients was conducted in order to make the "Pharmacy Vision for Patients" would be put into practice.

\section{New reimbursed program for pharmacist "Your pharmacist reimbursed program"2}

The Your Pharmacist Reimbursed Program (YPRP) has been established since April of 2016 for the services of outpatient pharmacy that Patient's family pharmacist conducted medication therapy management continuously and exclusively in cooperation with family physician. This YPRP consists of "Your Pharmacist Instruction Fee" and "Your Pharmacist Comprehensive Administrative Fee" as the fee reimbursed to a pharmacy with more strict and multiple requirements than those for the pharmacy that is reimbursed for the current medication therapy management fee (530 JPY ( $\fallingdotseq 4.8$ USD)). A pharmacy that meets requirements on "Your Pharmacist Instruction Fee" and got the written consent (Figure 1) from the patient may be reimbursed for $730 \mathrm{JPY}(\fallingdotseq 6.6 \mathrm{USD})$, and a pharmacy that meets requirements on "Your Pharmacist Comprehensive Administrative Fee" may be reimbursed for $2800 \mathrm{JPY}(\fallingdotseq 25.5 \mathrm{USD})$ in each patient prescription.

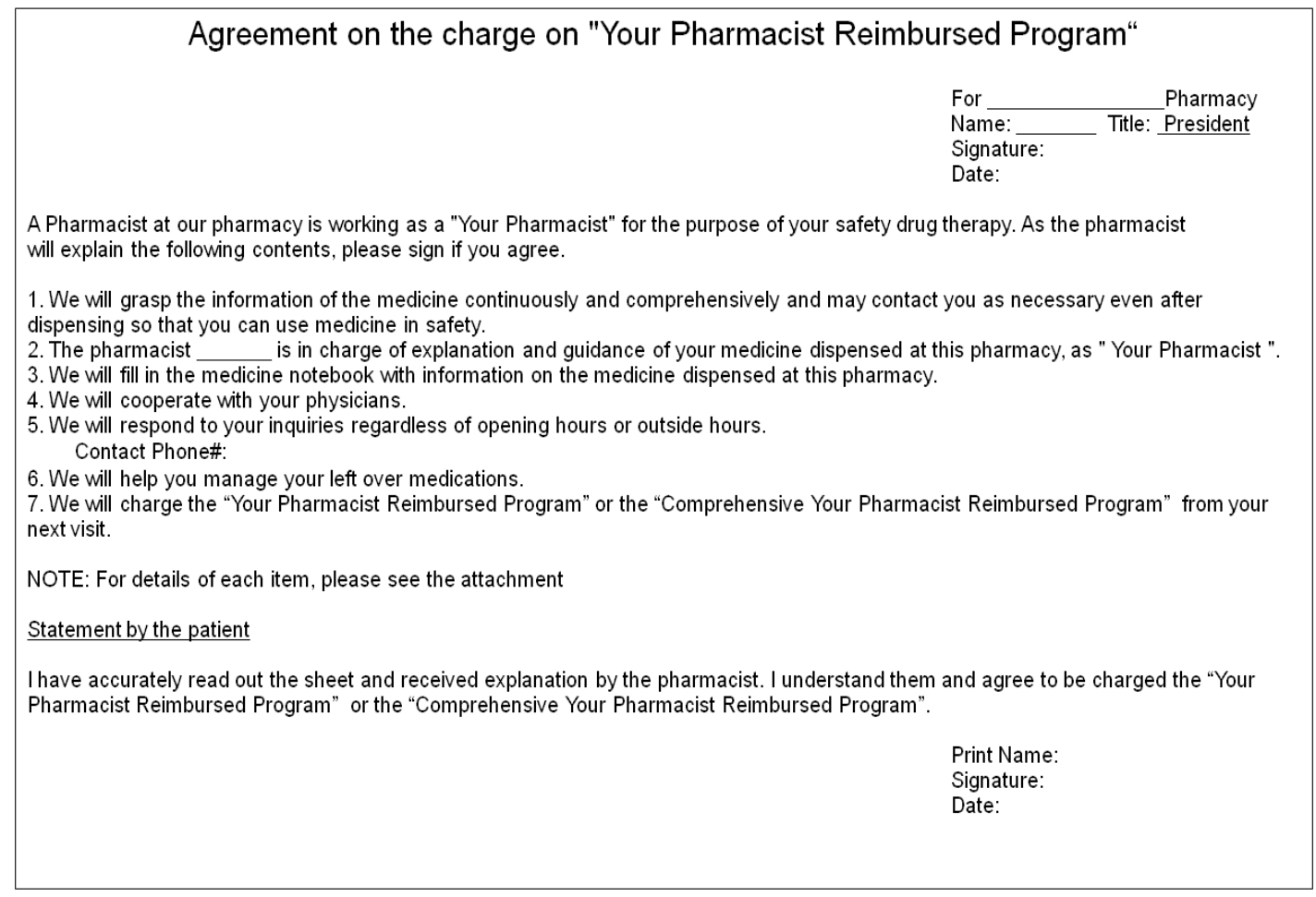

Figure I Example of the agreement on the charge on "Your Pharmacist Reimbursed Program".

When a pharmacy meets requirements on "Your Pharmacist Comprehensive Administrative Fee", the pharmacy may choose to be reimbursed for either of the two type of your pharmacist fee. But, when a pharmacy chooses to be reimbursed "Your Pharmacist Comprehensive Administrative Fee", the pharmacy may not be reimbursed for the dispensing fee and other fees related to a pharmaceutical management. "Your Pharmacist Instruction Fee" can be claimed for any patients, but "Your Pharmacist Comprehensive Administrative Fee" can be claimed only for the patients who are received the medical service in the Integrated Community Care System. The medical service in Integrated Community Care System is the comprehensive out-patient disease management framework, and, in the system, a family doctor specified by a patient manages the patient disease continuously and exclusively. The requirements on "Your Pharmacist Comprehensive Administrative Fee" consist of those on "Your Pharmacist Instruction Fee" and additional requirements that the pharmacy should provide information to the prescribing physician about the patient's medical compliance and effect/adverse events at every dispensing and should propose prescribing proposal as necessary.

\section{Requirement of YPRP}

The requirements on YPRP consist of those for the pharmacist operations and those for the pharmacist itself (Figure 2).The pharmacist reimbursed for the YPRP must gather information for all 
of the patient drug history and hospital/clinics visiting history, monitor the patient adherence and effect/adverse events and provide these gathered information to the patient's family physician if necessary, and also visit the patient's home to improve patient's adherence if necessary, in addition to usual medication therapy management services which included the documenting the drug history record, instructed on the use of drugs and providing the document for drug proper usage. Furthermore, the pharmacist have to inform the pharmacy opening hour, the pharmacist working hour to the patient, and also inform emergency contact number to the patients for 24 hours a day counseling services.

\section{Requirement of pharmacist operations}

1) Written consent from patient (see sample at Figure 1)

2) One pharmacist can claim the YPRP for one patient

3) Document the name of pharmacy and pharmacist on the patient's pocket drug history record

4) Conducting the medication therapy management which included the documenting the drug history record, instructed on the use of drugs and providing the document for drug proper usage

5) Correct and record the information for all of the patient drug history and hospital/clinics visiting history

6) Provide the counseling for 24 hours a day by the patient request

7) Monitor the effect/adverse effect of medicine and patients compliance and provide the these gathered information to the patient's family physician if necessary

8) Visit the patient's to improve patient's adherence if necessary

\section{Requirements of pharmacist itself}

The pharmacist to claim "Your Pharmacist Instruction Fee" should meet the followings:

1) More than three years working experience as pharmacist

2) Work more than 32 hours per week at the pharmacy where the pharmacist works

3) More than six month working experience at the pharmacy

4) Certified as continue professional development pharmacist

5) Participate the community activity for residents health improvement

Figure 2 Requirements of pharmacies and pharmacists for your pharmacist reimbursed program.

\section{Another fee related to YRRP}

Since the YPRP is fee in compensation for the pharmacist working as "Your Pharmacist", there were discussions about the needs for incentive of pharmacy to establish and maintain a good operation for the YRRP. Before introducing the YRRP, there were two kinds of add-on fee for in compensation for a good pharmacy dispensing operation. When introducing the YRRP at 2016, MHLW repealed the two kinds of add-on fee and introduced a new add-on fee called as "Conformity Dispensing Add-on Fee" of 320 JPY ( $\fallingdotseq 2.9$ USD) to maintain a good operation for the YRRP with restricts requirements in accordance with the Pharmacy Vision for Patients. After introducing the new add-on fee, some lobbyists strongly argued the re-assessment on the cost-effectiveness of the new add-on fee and argued pharmacy should contribute more community health management in a community health system. At 2018 reimbursement revision, MHLW updated "Conformity Dispensing Add-on Fee" to "Add-on Fee for Pharmacy Organization to Community Health Support" of 350 JPY $(\fallingdotseq 3.2$ USD) and updated the requirements (Figure 3 ). One of the key features in this update is that pharmacy should have achievements to demonstrate the pharmacy has an organization enough to contribute the community health management when pharmacy asks "Add-on Fee for Pharmacy Organization to Community Health Support". The required achievements are so challengeable that pharmacy should make more effort to contribute the community (Figure 4).

\section{Impact of YPRP on pharmacists and patients}

The MHLW conducted the survey for the effect and impact of YPRP on pharmacies and patients ${ }^{3}$ as a randomized survey for 1000 pharmacies that operated the YPRP and 1000 pharmacies that operated no YPRP by mailing the questionnaire (collection rate was $57.4 \%$ ) and as a systematic sampling survey for first and second patients who visited the pharmacies during the survey period of each pharmacies by handing the questionnaire and mailing back to MHLW directory (1424 responded).

\section{Impact and effect of YPRP to the care of pharmacist}

The result of the survey for the pharmacies that operated the YPRP on the effect of YPRP to the care of pharmacist (Figure 5) appears that the YPRP affects positively the care of pharmacist, especially at "Pharmacists became to assess multiple medication and medicine interaction more easily." "Pharmacy became to provide patient compliance instruction conscientiously" and "Pharmacy became to work more on decreasing on leftover medications." The survey result also shows the YPRP affects positively the relationships with patient at "Frequency of a consultation from patients or their family had been increased." "Patients became to bring multiplicate prescriptions written not by only one clinics but a number of clinic" and "Patients' medication compliance had been improved." The YPRP seems to enable the pharmacist to provide more appropriate care to the patients with more trust from the patients and their family. On the other hand, the survey result shows the YPRP makes few effects on the relationship with physicians at "Frequency of providing information on patient's adverse event and medication compliance to the physician had been increased" and "Frequency of a suggestion on a prescription appropriateness for the physician had been increased." It seems that the effects of YPRP on the cooperation with physicians in pharmacological management are very little. 
(1) Pharmacy should have achievements to demonstrate that the pharmacy has an organization enough to contribute the community health management (Fig 3-2) .

(2) Pharmacy should provide an appropriate medication therapy management and medication instruction for each patient.

(3) Pharmacy should provide the appropriate information on drugs related to medication according to patient's request.

(4) Pharmacy should open for more time enough to provide appropriate pharmacy service*

(5) Pharmacy should stockpile a sufficient number of medicines**.

(6) Pharmacy should have established appropriate organization and function to provide necessary proper pharmaceutical management and medication instruction and should have provided the information on the pharmacy organization to a home care service ${ }^{* * *}$.

(7) Pharmacy should have established a system to dispense drugs and provide pharmaceutical management/medication instruction for athome patients for 24 hours only by the pharmacy individually or by the group of the pharmacy and the other neighboring pharmacies.

(8) Pharmacy should have established the cooperation system with clinics or hospitals and visiting nursing stations related to support for home care in the area.

(9) Pharmacy should have established the coordination system with coordinators with other health care services and welfare services in the area.

(10) Pharmacy should have the past records that the pharmacy contribute patients' safety ${ }^{\star * * *}$.

(11) The proportion of the unit quantity based amount of generic drugs dispensed at the pharmacy to the amount of drugs except for brandname drug with no generic drug should be $50 \%$ or more, if the proportion of dispensing by prescription issued by a specific medical institution exceeds $85 \%$.

Figure 3 Requirements of "add-on fee for pharmacy organization to community health support."

*The interpretation of "open more time enough to provide appropriate pharmacy service" is "open for more than 45 hours per a week, for more than 8 hours per a day on weekday and open on Saturday or on Sunday".

**The interpretation of "a sufficient number of medicines" is "more than I,200".

***The interpretation of "appropriate organization and function" is "the manager of pharmacy has more than five years working experience as pharmacist and more than six month working experience at the pharmacy and works more than 32 hours per week at the pharmacy"," the pharmacy has construction such as partition to protect the patients privacy", "the pharmacy has systems for continue education for pharmacists", "The pharmacy has function to collect the Drug Information" and " the pharmacy display that the pharmacy conduct the health consultation"

*****The interpretation of "achievements that the pharmacy contributes patients' safety" is "pharmacy has cases of preavoid such as prescription questions prevent from adverse events and/or not-intended drug efficacy and has a standard operating procedure on adverse event reporting."

Pharmacy has the past records on the number of times per a full-time pharmacist regarding the all of the followings;

(1) $\geqq 400$ times of a patient reception while pharmacy-off-hour

(2) $\geqq 10$ times of a drug therapy management on narcotic drugs

(3) $\geqq 40$ times of a prescription question to prevent from duplicate administration and/or drug interaction

(4) $\geqq 40$ times of the YPRP

(5) $\geqq 12$ times of a special support for the patients to have difficulty in taking medicine

(6) $\geqq 1$ times of an integration of a plural internal medicines

(7) $\geqq 12$ times of a drug therapy management for a homecare patient who is not at a care home.

$(8) \geqq 60$ times of monitoring patient's compliance and providing drug information after a patient go back to the home

Figure 4 Achievements to demonstrate that the pharmacy has an organization enough to contribute the community health management.

At the free comments in the survey, there are comments for relationships with patients such as the opportunity to talk with patients was increased, pharmacist could talk with patients for their personal issues, pharmacists could give smooth consultation on the medication compliance and the complaints from patients was decreased. It seems that the relationships between pharmacist and patient are closer and improved. The survey result also says that pharmacist received the opinions from patients such as "Feeling of sense of safety and secure to have medicine from having own pharmacist and 24 hours a day counseling services". It seems that the YPRP can provide positive effect on drug therapy and that the patients reliance on pharmacists by providing appropriate and close consultation on drug therapy from pharmacist. However, there are negative comments from pharmacist, for example, the burdens on more contact from patients at off hours such as frequent call from patient at night was increased.

Furthermore, the result of the survey on the effect of frequency of a prescription question with comparison between the YPRP and usual medication therapy management services (Figure 6) shows the YPRP's prescription question rate was $9.2 \%$ which is three times bigger than traditional medication therapy management services (3.0\%). The prescription question based on the pharmacological skills 
also seems to be increased that $58 \%$ of YPRP's against the $53 \%$ of traditional medication therapy management services. According to the survey on pharmacist's expectation for YPRP at Figure 5, pharmacist's expectation that YPRP produces increasing prescription questions is a little positive as compared with other expectations. But, according to Figure 6, the actual number of prescription questions has been increased. These results suggest YPRP makes a positive effect on pharmacists to do more prescription question not only as their attitude but also as their actual performance. It seems that improvement of the communication between pharmacists and patients by the YPRP gives pharmacist opportunities to make questions on the drug therapy management and then pharmacists become to have more communication with physicians.

\section{Patient's intention for family pharmacist}

The patient expectations for family pharmacists were surveyed with multiple answers with patients who received YPRP services or with traditional medication therapy management services (Figure 7). "My family pharmacist confirms all my medication together prescribed by plural prescribers", "My family pharmacist comprehends all kinds of my medications." "My family pharmacist explains my medication therapy with easy-to-understand" and "My family pharmacist corresponds to my medical consultation." were mostly selected as patient expectations for family pharmacist by both patients, though, it seems that the patients receive the YPRP services show more expectations for family pharmacist.

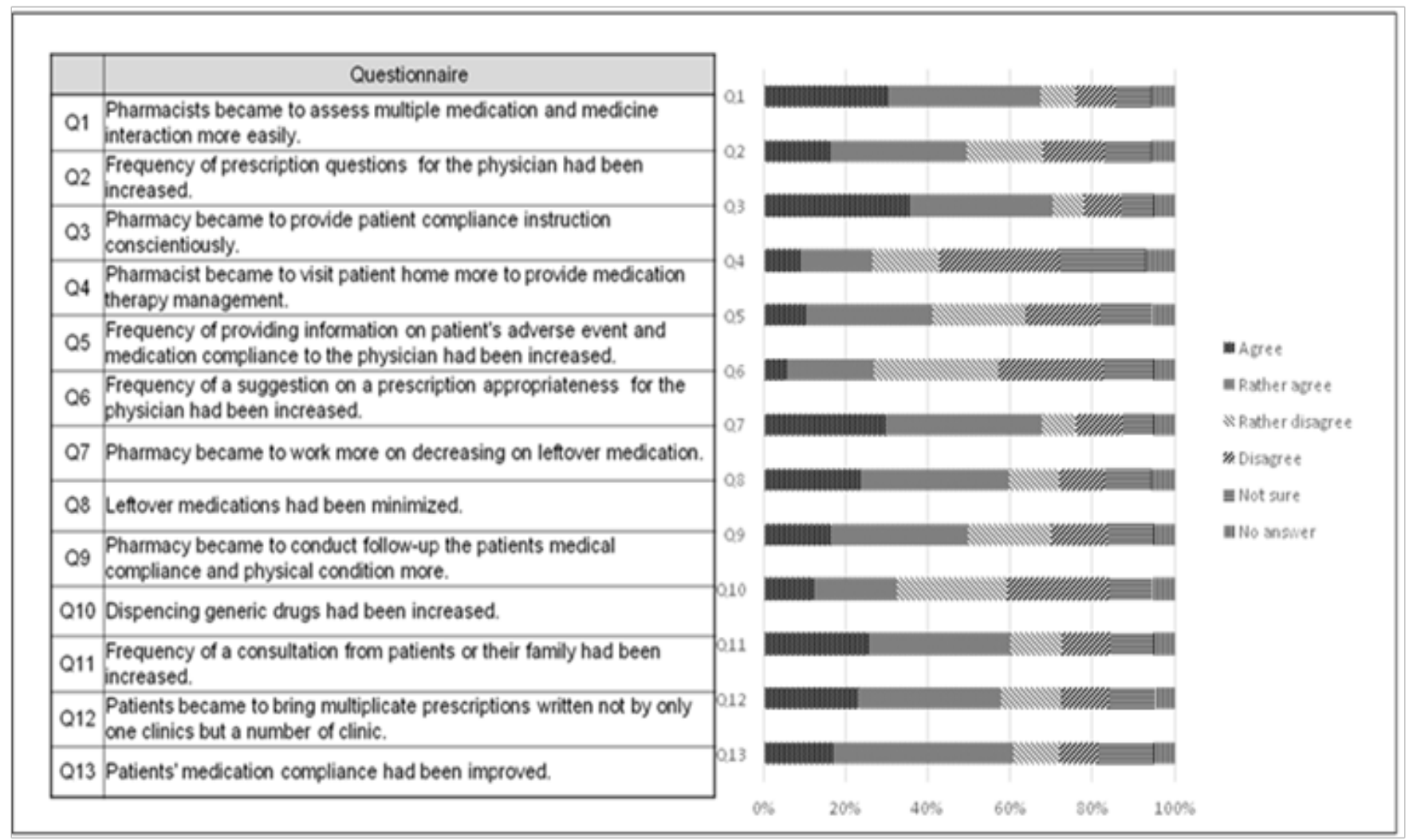

Figure 5 Impact and effect on the care by pharmacist YPRP.

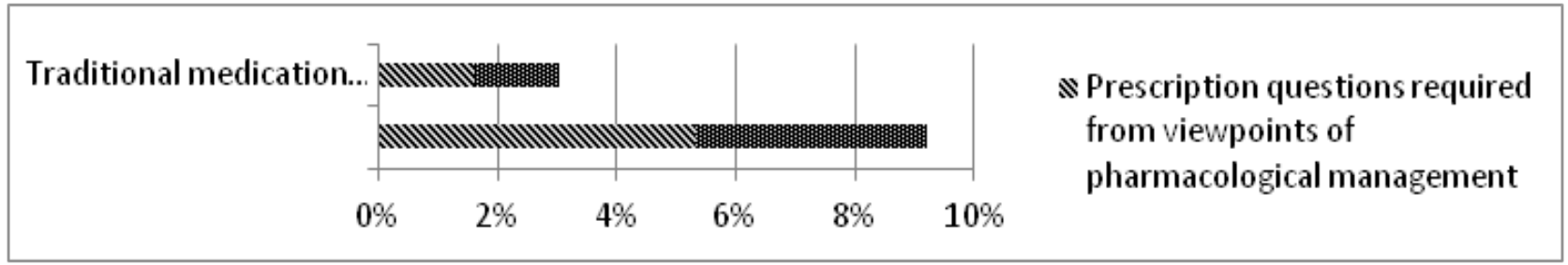

Figure 6 Frequency of prescription question. 


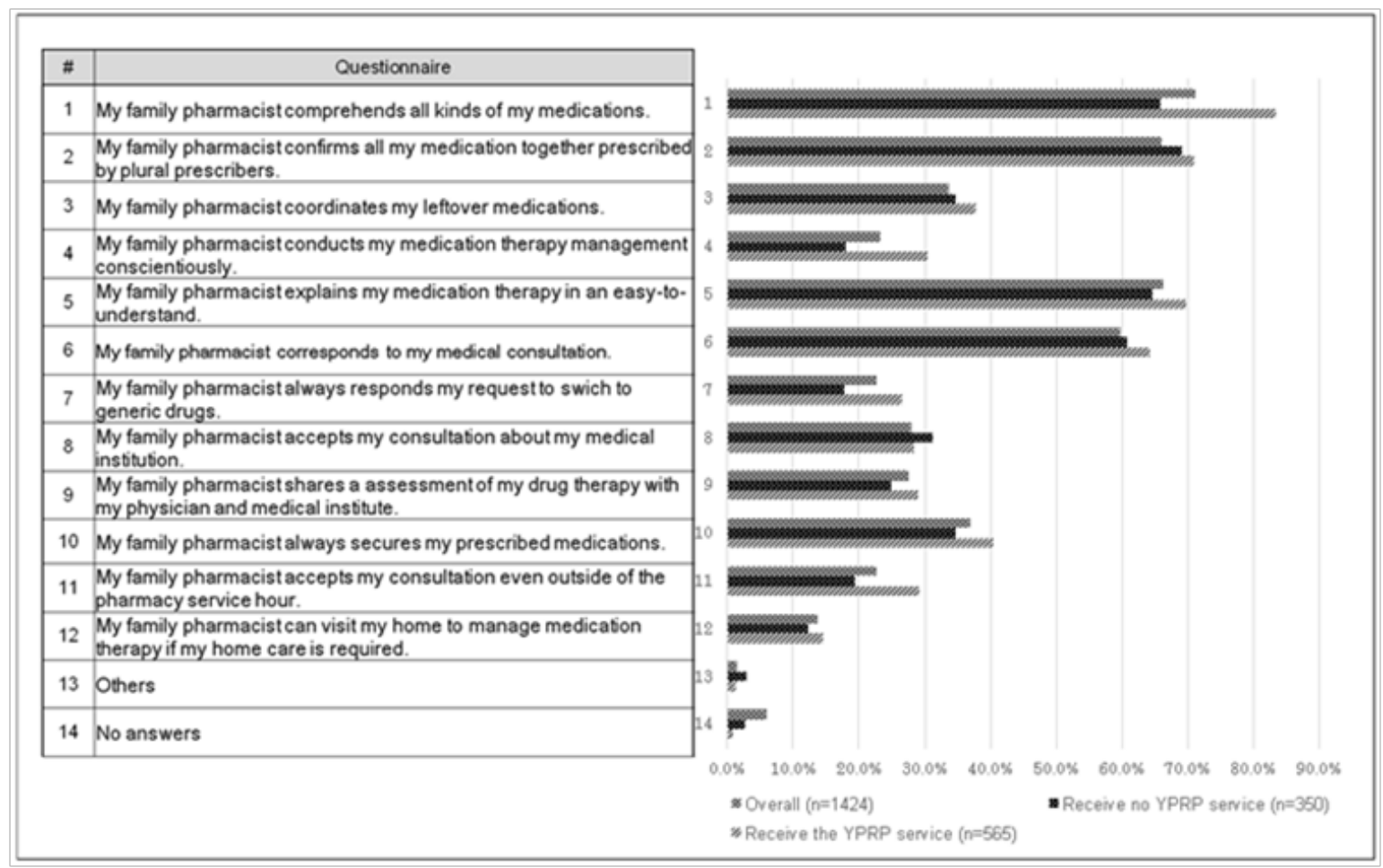

Figure 7 Patient's satisfaction and expectation for their family pharmacist.

\section{Conclusion}

The MHLW has announced the new policies to enhanced pharmacists, pharmacies to play the pivotal role for medication therapy management of patients in Japanese aging society and also established the YPRP for that pharmacist will be able to play expected role in providing the care to the patients. From the survey for the effect and impact of YPRP conducted by MHLW, introducing the YPRP has seen that relationship between pharmacists and patients has been closer and improved, and also pharmacist could provide medication therapy management to the patient continuously and exclusively. While for patients, change in consciousness has been founded that expectation for family pharmacist is growing that family pharmacists could observe all taken medications for each patient. It seems that the YPRP gave good effect and impact to the medication therapy in Japan. However, we don't have so many knowledge with YPRP since the YPRP has been into effect since 2016, we should improve the YPRP in order to increase the quality of care from pharmacist and also to optimize the medication therapy in Japan. Therefore, we need more survey and research to improve the YPRP. Pharmacies/pharmacists needs to change the function and role of pharmacy to meet patient needs and demands from public society with using the new policies which is introduced by the MHLW. Furthermore, pharmacies/pharmacists have to use the newly established YPRP in careful manner and improve the satisfaction of patients, and then, pharmacies/pharmacists have to step up to the next stage to adapt to the progressive aging society by expanding and developing the YPRP. It could say that The YPRP is the first step to cope with the progressive aging society at 2025 .

\section{Acknowledgements}

None.

\section{Conflict of interest}

The author declares that there is no conflict of interest.

\section{References}

1. Pharmacy Vision for Patients. Japan: Ministry of Health, Labour and welfare; 2014.

2. The revision of Pharmacy reimbursement program. Japan: Ministry of Health. Labor and Welfare; 2016.

3. Leftover, duplicate and polypharmacy survey for medication proper use and survey for effect and status of implementation of revision of pharmacy dispensing fee including evaluation of the Your Pharmacist Reimbursed Program. China: Chinese Medical Association; 2010. 